\title{
Simulating Remote Support for Mathematical Perseverance Through a Digital Sketching Application
}

\author{
Joseph DiNapoli \\ Montclair State University \\ Malack Amenya \\ Montclair State University \\ Lelli Van Den Einde \\ University of California, San Diego \\ Nathan Delson \\ University of California, San Diego \\ Elizabeth Cowan \\ eGrove Education, Inc.
}

\begin{abstract}
This exploratory study showed how a digital sketching application helped keep $4^{\text {th }}$-grade students engaged with challenging mathematics tasks and support their perseverance to learn mathematics conceptually. The application is currently being developed as a tool based on digital assignments for which students freehand sketch visual representations to solve conceptual fractions tasks. Participants engaged with a simulation of the application and received personalized and conceptual feedback based on their sketching mistakes. Our findings showed that participants were able to leverage such feedback to persevere with the task and make mathematical progress, even at moments when they were most challenged and frustrated.
\end{abstract}

Keywords: perseverance, engagement, digital technology, mathematics education

\section{INTRODUCTION AND BACKGROUND}

Perseverance, or initiating and sustaining in-the-moment productive struggle in the face of one or more obstacles, setbacks, or discouragements, is a constructive process by which understandings are developed (DiNapoli \& Miller, 2020). Opportunities to learn emerge as one encounters content with which they are unfamiliar, and resiliently working to overcome such obstacles is central to making sense of a new idea. Perseverance is different from simple engagement or time-on-task; perseverance implies an encounter with a setback that spawned productive new efforts that pay dividends toward learning (DiNapoli \& Miller, 2020). Reconciling times of substantial uncertainty (i.e., answering a problem incorrectly) may be most critical for learning. The processes of struggle to approach, reach, and make continued progress despite a 
problem-solving misstep puts forth cognitive demands upon the learner that are conducive for development of conceptual ideas (Zaslavsky, 2005). These ideas have been echoed for learning mathematics because students develop their mathematical knowledge, both in content and ways of thinking, through productive struggle, or as they grapple with mathematical ideas that are within reach, but not yet well formed (Hiebert et al., 1996). Consequently, recent mathematics education research (DiNapoli, 2019a, 2019b; DiNapoli \& Miller, 2020; Morales, Jr. \& DiNapoli, 2018) have explicitly attended to nurturing perseverance in the classroom, namely by providing students consistent "opportunities and supports to engage in productive struggle as they grapple with mathematical ideas and relationships" (NCTM, 2014).

In K-8 classrooms, the meaning of rational numbers (i.e., fractions) is a domain rich with opportunities to support student perseverance. A fraction is a type of number that can be expressed in different ways: numerator over denominator (e.g., 3/4), decimal notation (e.g., 1.7), or whole number followed by a number less than 1 written as a fraction (e.g., 2 1/5). For decades, fractions have been known as "... undoubtedly the most challenging number system of elementary and middle school mathematics" (Kilpatrick, Swafford, \& Findell, 2004). This point applies to both learning and teaching. The challenge of making sense of fractions stems from curricular, pedagogical, and epistemological factors, with typical American curriculum and instruction over-emphasizing the part-whole way of understanding (Maher \& Yankelewitz, 2017). Related, students rarely have consistent opportunities to explore the distinct meanings of fractions concepts, such as the meanings of the numerator and denominator, operator interpretations, the meaning of ratios, and how fractional quantities can be constructed by partitioning and iterating from reference quantities

One classroom activity that attends to such exploration of fraction concepts is student sketching of visual representations. When students sketch models of fraction concepts and operations, they have the opportunity to grapple with various ways of interpreting fractions (Steffe \& Olive, 2010). This includes understanding fractions as the result of dividing two numbers, as the ratio of two quantities, as operators, as measures, and as parts of wholes or parts of sets. The act of freehand sketching visual representations has been shown to help students develop conceptual understandings, problem-solving, logical reasoning, and mathematical discovery (Arcavi, 2003). Moreover, an incorrectly drawn sketch can give insight into the thought process behind the student's mistake which could be used by a teacher to support productive struggle. Several cognitive and neurological studies of mathematical thinking have shown the importance of engaging with and creating visual representations for mathematics learning (Boaler et al., 2016; Hubbard et al., 2005). This neurological evidence, coupled with student development research, drives the accelerating position that "mathematics teaching and learning needs to become more visual" (Boaler et al., 2016). This combination of learning through engagement with visual representations and freehand sketching underpins an effective practice for mathematics learning, especially in the context of fractions.

Yet, for students to persevere with such challenging sketching fraction tasks they need ample support from skilled teachers. This support includes monitoring student thinking closely to provide differentiated feedback at moments when students are on the verge of giving up. Differentiated feedback can be framed as levels of scaffolding, ranging from developing low-level conceptual thinking scaffolds to medium-level explaining, reviewing, and restructuring scaffolds, to high-level environmental provisioning (Anghileri, 2006). Such scaffolding can also be gentle reminders for students to attend to the connections between the big ideas at play in a mathematical situation. Scaffolding is often most effective when it is personal to the actual engagement of the student. Personalized feedback has been shown to support mathematics achievement and help students rely less on memorizing procedures and more on their own thinking to persevere with a challenging mathematics task (Freeburn \& Arbaugh, 2017; Zhang et al., 2015). However, consistently providing attentive support for all students at specific moments of struggle logistically difficult for teachers in face-to-face educational settings, and even more difficult for teachers working in remote educational settings.

Incorporating intelligent digital technology can help with this transition to remote learning by helping students engage with challenging mathematics without their teacher present. However, many mathematics education applications tend to rely on routine-based learning and are absent of opportunities for concept development through supported productive struggle. While some mathematics education applications are 
built on educational research to promote differentiated learning (e.g., Amplify Fractions, Teachley, DoodleMaths), few of them offer personalized mathematical feedback to help keep students engaged with challenging ideas. Most importantly, no current mathematics education application incorporates freehand sketching and personalized feedback to support conceptual learning and perseverance.

In all, the purpose of this exploratory study was to test the features of a digital sketching application called Drawn2Math on its efficacy to remotely support student perseverance with sketching-based fractions tasks. This research addresses the current needs of mathematics education, especially related to keeping students mathematically engaged during the COVID-19 pandemic. The sections that follow detail our methodology, results, and conclusions.

\section{METHODOLOGY}

Our chief methodological concern was to engage students with features of the Drawn2Math application - a software application being designed to leverage automatic grading algorithms and predefined mathematical feedback to support student perseverance with sketching-based fraction tasks. However, at the time of study the Drawn2Math software was still in the development stage, therefore we engaged students in a simulation of the application to study the effectiveness of the overall approach. We used a Wizard of Oz approach (see 2.2 Data Collection below) to simulate many salient features of Drawn2Math's. These features undergird the development philosophy of Drawn2Math as an application that could perform like an expert teacher looking over the shoulder of each student that will provide just the right amount of guidance to support the conceptual learning of mathematics.

The final version of the Drawn2Math application will contain several modules of research-based sketching-based fraction tasks, including sketching fractions as measurements and sketching the actions of fraction operations. We chose to focus on fractions tasks due to the richness of information in student sketches and the importance of strong conceptual understanding of rational numbers in the K-8 curriculum. Drawn2Math will be equipped with a digital sketching platform capable of interpreting student-drawn conceptions of fractions and providing meaningful feedback to support perseverance. The Drawn2Math grading algorithms will use partially completed or incorrect sketches to identify students' conceptual errors. These identified errors will be compared to common mistakes and thereby trigger personalized mathematical feedback that meets the child at their current stage of thinking. This feedback is designed to support students to be resilient and continue to persevere with their problem-solving. Such feedback will be predefined, personalized based on the mathematical mistake made, and informed by scaffolding research. Additionally, students will be able to use different colored pens for their scratch work and final answers, encouraging exploration and problem-solving while also providing the algorithm further insights into the student thought process. Through this platform and feedback system, we will be better able to understand how a child is thinking about mathematics and better able to support their continued mathematical progress. Examples of how the Drawn2Math digital sketching platform and feedback system will work are presented in the Results.

\section{Participants}

The participants for this study were 10 fourth-grade students from the Mid-Atlantic region of the United States. Each participant was from the same fourth grade class with the same teacher and was learning about fractions for the first time. All participants were purposely chosen to have demonstrated, via pretest, that they had not yet learned or mastered certain fraction concepts. In this study, approximately 6 hours of student work were evaluated.

\section{Data Collection}

To collect data, each participant engaged with a simulation of the Drawn2Math application. During the simulation, participants thought aloud as they problem-solved with up to 18 sketching-based fraction tasks on a touchscreen device and received personalized mathematical feedback when necessary. This feedback varied, depending on the mathematical mistake, and was informed by scaffolding research. Participants' 
activity on the touchscreen device was video recorded, the student's voice was audio recorded, and field notes of the simulation were taken. To simulate the application, we used a Wizard of Oz approach (Kelley, 1984). The Wizard of Oz approach is a usability trial in which a participant interacts with an unfinished system or product while a researcher ("the Wizard") simulates the behavior of the system. In our case, participants used an iPad sketching program, Pixelboard, through which a remote researcher (the first author) could see in real-time what they are drawing. Thus, each participant worked on their sketching task individually and were monitored by the researcher remotely. The researcher's job was to act as the Drawn2Math grading algorithm - to administer predefined and personalized mathematical feedback when participants submitted a certain incorrect sketch, or to mark a submission correct and advance the participant to a new task. The Wizard's feedback was only via windows of text and/or images that appeared on the participant's iPad. The Wizard did have a line of sight to the participant so they could observe body language. Participants were informed that they were allowed to stop working at any time.

\section{Data Analysis}

We analyzed the data to discern the frequency and rate at which participants were persevering as they engaged with the simulated Drawn2Math application. We used the Three-Phase Perseverance Framework (3PP) (DiNapoli \& Miller, 2020) (see Figure 1) as a qualitative analytical tool. The 3PP has been used in research to operationalize perseverance in problem-solving (DiNapoli, 2019a, 2019b; DiNapoli \& Miller, 2020; Morales, Jr. \& DiNapoli, 2018). It was designed to reflect perspectives of concept (Middleton et al., 2015), problem-solving actions (Pólya, 1971), self-regulation (Carver \& Scheier, 2001), and making and recognizing mathematical progress (Gresalfi \& Barnes, 2015). The three-phases of the 3PP are (1) the Entrance Phase, which considers if the task at hand necessitates perseverance, (2) the Initial Attempt Phase, which considers if a participant decided to initiate and sustain their effort toward a task solution and if those efforts were mathematically productive, and (3) the Additional Attempt Phase(s), which considers if a participant decided to re-initiate and re-sustain their new effort toward a task solution after a substantial setback(s) and if those new efforts were mathematically productive. A participant could theoretically experience multiple Additional Attempt Phases if they encountered multiple substantial setbacks while working.

\section{FIGURE 1 \\ THREE-PHASE PERSEVERANCE FRAMEWORK (3PP)}

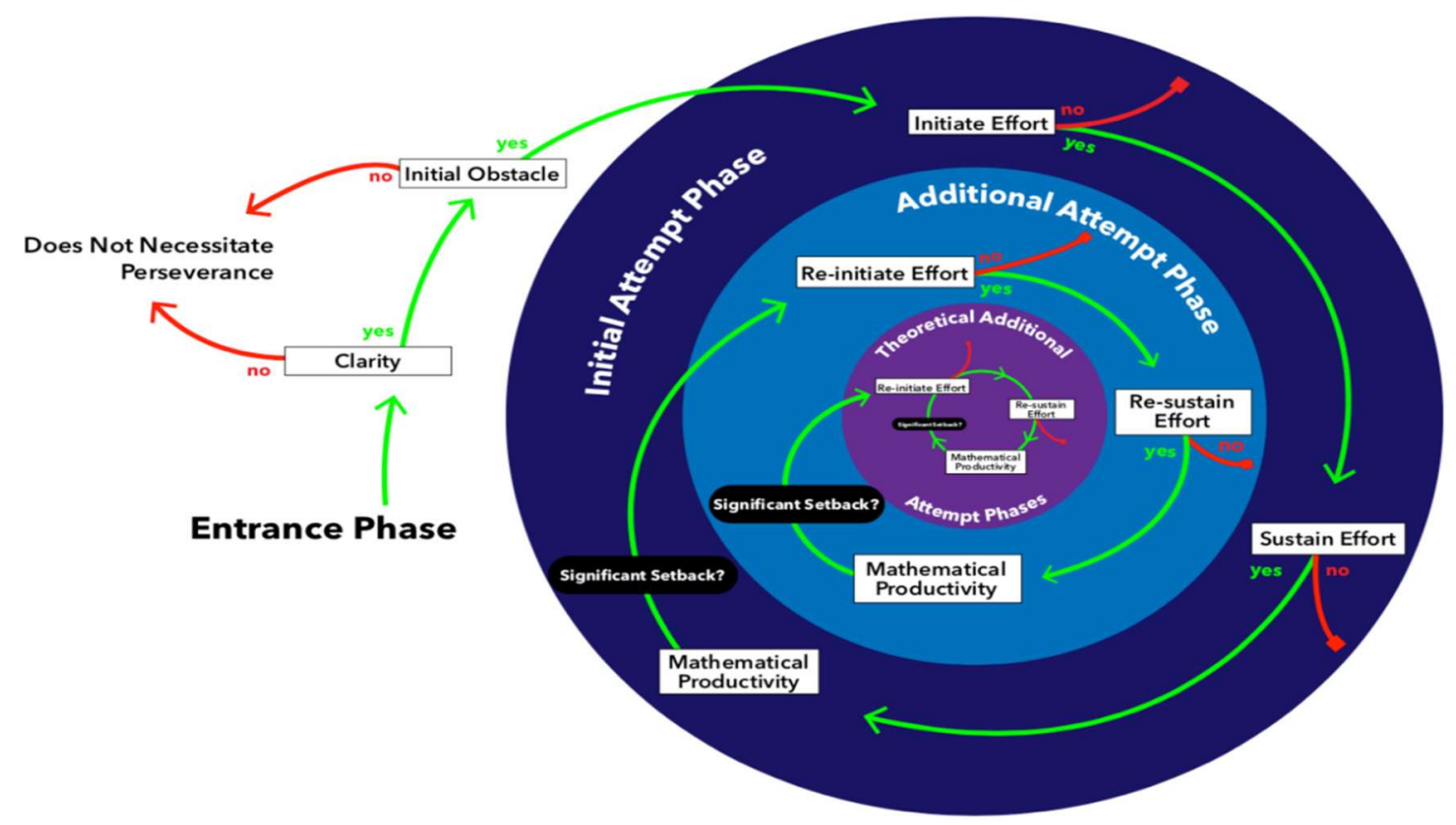

44 Journal of Higher Education Theory and Practice Vol. 21(4) 2021 
In this study, we were interested in participants' perseverance after they submitted an incorrect sketch and subsequently received personalized mathematical feedback. Therefore, our analysis focused on participants' Additional Attempt Phases. This implies that participant data for tasks solved during an initial attempt, and thus participants never submitting an incorrect sketch, were not considered in this paper. We defined a substantial setback as moments when a participant submitted a mathematically incorrect answer while working on a Drawn2Math sketching-based task and subsequently received personalized feedback. We defined mathematical productivity as submitting a correct answer or as demonstrating progress toward better understanding a mathematical idea compared to previous evidence of understanding. Thus, our analyses considered the frequencies of participants' substantial setbacks and coinciding receival of personalized mathematical feedback (denoted as A), the frequencies of participants' re-initiated and resustained new efforts after substantial setbacks (denoted as B), and the frequencies of participants' mathematical productivity as a result of re-initiated and re-sustained new efforts (denoted as $\mathrm{C}$ ).

We report about two constructs in our results: the Reengagement Rate (RR) and the Perseverance Success Rate (PSR). Both of these constructs stemmed from the foci of our analyses. The Reengagement Rate captured participants' willingness to reengage with the task and not give up after submitting a mathematically incorrect answer for a Drawn2Math sketching-based task. This describes the resilience of a participant after a substantial setback and receival of subsequent personalized mathematical feedback. Thus, the Reengagement Rate was calculated as the quotient of the frequency of participants' re-initiated and re-sustained new efforts after substantial setbacks and the frequency of participants' substantial setbacks, or $\mathrm{RR}=\mathrm{B} / \mathrm{A}$. The Perseverance Success Rate captured participants' mathematical productivity as a result of their willingness to reengage with the task and not give up after submitting a mathematically incorrect answer for a Drawn2Math sketching-based task. This describes participants' productive struggle after a substantial setback and receival of subsequent personalized mathematical feedback, that is, that participants' resiliency paid dividends of mathematics learning. Thus, the Perseverance Success Rate was calculated as the quotient of frequency of participants' mathematical productivity as a result of re-initiated and re-sustained new efforts and the frequency of participants' substantial setbacks, or PSR = C/A. In sum, the Reengagement Rate and the Perseverance Success Rate both capture evidence of resiliency in participants' engagement, but the Perseverance Success Rate alone captures the mathematical productivity of such resilience.

\section{RESULTS}

In this section, we first describe participants' perseverance working on Drawn2Math sketching-based tasks, after they submitted an incorrect sketch and subsequently received personalized mathematical feedback. Recall, we only considered participants' experiences with tasks on which they made mistakes participants' experiences with tasks on which they made no incorrect submissions were not considered in our perseverance analysis. Additionally, recall that participants were working on these tasks alone during the Drawn2Math simulation, without a teacher present. We report participants' collective Reengagement Rate and Perseverance Success Rate as findings. Second, to illustrate these findings, we present one representative participants' experience working on a sequence of three related sketching-based tasks.

\section{Participants' Perseverance on Sketching-Based Tasks}

In general, our findings showed that participants working on challenging Drawn2Math sketching-based tasks were often able to leverage the personalized mathematical feedback to reengage and persevere with the task, even after making substantial mistakes. See Table 1 for more details of these findings. While working on up to 18 sketching-based tasks, all 10 participants encountered substantial setbacks by submitting at least one incorrect answer. The frequencies of incorrect submissions varied by participant, with the minimum number of incorrect submissions being two and the maximum number of incorrect submissions being 19. Across all participants, the total number of incorrect submissions was 65. Recall that personalized mathematical feedback accompanied each incorrect submission. Thus, participants received 
personalized mathematical feedback 65 times after each incorrect submission. This feedback varied, depending on the mathematical mistake.

TABLE 1

PERSEVERANCE ON SKETCHING-BASED TASKS FOR WHICH THE INITIAL
SUBMISSION WAS INCORRECT

\begin{tabular}{c|c|c|c|c|c}
\hline \hline & $\begin{array}{c}\text { Incorrect } \\
\text { Submissions }+ \\
\text { Personalized } \\
\text { Feedback (A) }\end{array}$ & $\begin{array}{c}\text { New Efforts } \\
(\boldsymbol{B})\end{array}$ & $\begin{array}{c}\text { Productive } \\
\text { New Efforts } \\
(\boldsymbol{C})\end{array}$ & $\begin{array}{c}\text { Reengagement } \\
\text { Rate (RR) }\end{array}$ & $\begin{array}{c}\text { Perseverance } \\
\text { Success Rate } \\
\text { (PSR) }\end{array}$ \\
\hline Participant 1 & 19 & 17 & 14 & $89 \%$ & $74 \%$ \\
\hline Participant 2 & 2 & 2 & 2 & $100 \%$ & $100 \%$ \\
\hline Participant 3 & 2 & 2 & 2 & $100 \%$ & $100 \%$ \\
\hline Participant 4 & 11 & 11 & 9 & $100 \%$ & $82 \%$ \\
\hline Participant 5 & 7 & 7 & 7 & $100 \%$ & $100 \%$ \\
\hline Participant 6 & 7 & 5 & 5 & $71 \%$ & $71 \%$ \\
\hline Participant 7 & 9 & 9 & 8 & $100 \%$ & $89 \%$ \\
\hline Participant 8 & 2 & 2 & 2 & $100 \%$ & $100 \%$ \\
\hline Participant 9 & 3 & 3 & 3 & $100 \%$ & $100 \%$ \\
\hline Participant 10 & 3 & 3 & 3 & $100 \%$ & $100 \%$ \\
\hline Totals & $\mathbf{6 5}$ & $\mathbf{6 1}$ & $\mathbf{5 5}$ & $\mathbf{9 4 \%}$ & $\mathbf{8 5 \%}$ \\
\hline \hline
\end{tabular}

Legend: Reengagement Rate (RR) = B/A; Perseverance Success Rate (PSR) = C/A

After receival of personalized mathematical feedback, participants made a new effort by re-initiating and re-sustaining their effort toward a task solution $94 \%$ of the time, as noted by the Reengagement Rate in Table 1. This suggests that participants were motivated by the personalized mathematical feedback to stay engaged with the mathematics, despite a substantial setback. Related, after receival of personalized mathematical feedback, participants were mathematically productive in their new effort toward a task solution $85 \%$ of the time, as noted by the Perseverance Success Rate in Table 1. This suggests that not only did the personalized mathematical feedback help participants to stay engaged with the task despite a substantial setback, but such feedback supported them to productively struggle to make continued mathematical progress. Many participants encountered several substantial setbacks across their work with a challenging sketching-based task(s); it follows that they received several pieces of personalized feedback that helped them resist the urge to give up and manage their struggle and frustration over time. Further, the $85 \%$ Perseverance Success Rate suggests that this continued engagement paid dividends for these participants in terms of their mathematics learning.

\section{Illustrative Case: Jamie's Experience With Sketching-Based Fraction Tasks}

To illustrate the ways in which mathematical perseverance was supported remotely, consider Jamie's (pseudonym) (Participant 4 in Table 1) experience with a subset of the sketching-based tasks in her Drawn2Math simulation. Since participants' activity on the touchscreen device was video recorded, and since participants were thinking aloud as they worked remotely, we are able to share samples of student work accompanied by quotes of their think-aloud. We describe Jamie's experience with three related tasks in which she asked to draw a rectangular area model to represent a fractional quantity relative to a given fractional quantity. We chose to report on these three tasks because they necessitated perseverance for Jamie. Such tasks are often challenging for students learning about fractions for the first time because they must reconcile the idea that the given quantity is not one-whole, and also because of the dependent relationship between the prompted quantity and given quantity. 
First consider Task I, which prompted Jamie to draw an area model of $2 / 3$ given a rectangular area model representing 1/3. The given model of $1 / 3$ had an area of 6 square units ( 2 units by 3 units). Jamie initiated her effort toward a task solution by saying "This is one is for $2 / 3$ " and sustained her effort by sketching a rectangle with an area of 4 square units ( 2 units by 2 units) as her answer, which was incorrect. It seemed she was sketching two-thirds of the given rectangle. She submitted this answer and it was graded as incorrect, marking her first substantial setback $(\mathrm{A}=1)$. Jamie exclaimed, "Oh no!" as her answer was graded as incorrect, suggesting her disappointment. The nature of her incorrect answer initiated her first piece of personalized feedback, which said "The size of your $2 / 3$ is incorrect" (see Figure 2, left). Jamie's mistake was likely rooted in her not recognizing that the given rectangle was representing a quantity of $1 / 3$, not 1 whole. Thus, her piece of lower-level scaffolded feedback was designed to gently remind her refocus her attention on the given information.

FIGURE 2

\section{JAMIE'S EXPERIENCE ON TASK I}
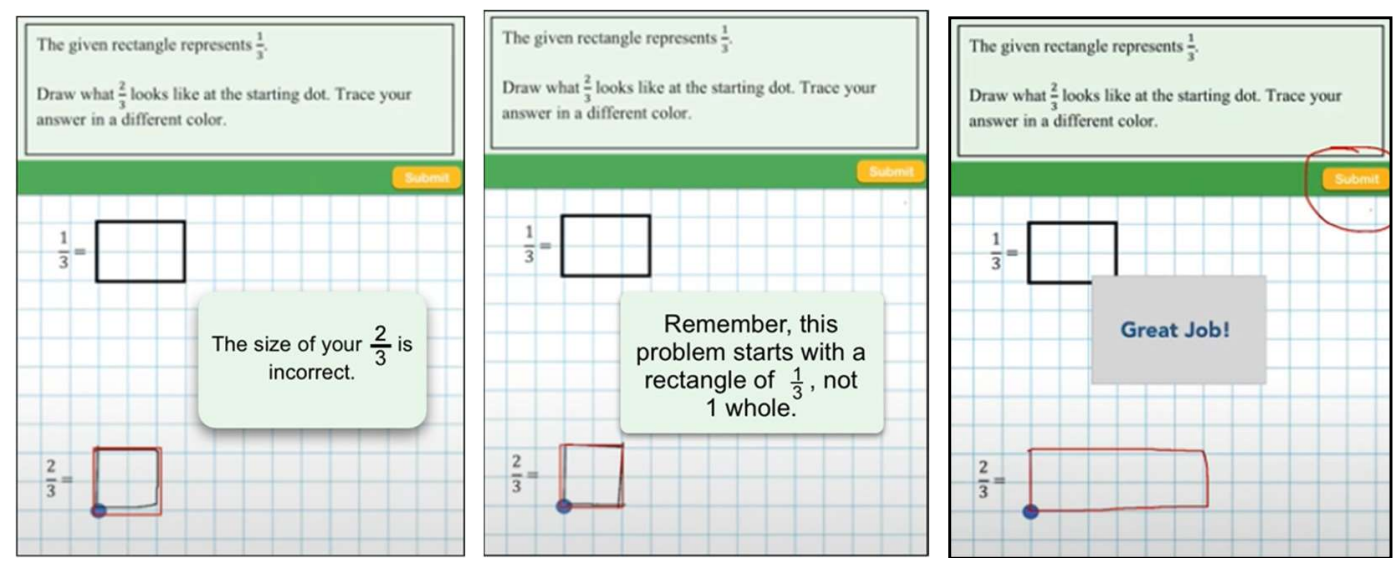

Jamie responded resiliently once she received this feedback and decided to re-engage to make a new effort toward a task solution $(B=1)$. Jamie re-initiated her new effort by saying " $\mathrm{Hmm}$, I have to draw the $2 / 3$ " and re-sustained her effort by sketching a rectangle with an area of 4 square units ( 2 units by 2 units) as her answer once again. Since this was the same answer as her previous incorrect submission, this new effort was determined to be not mathematically productive $(\mathrm{C}=0)$. She submitted this answer and it was again graded as incorrect, marking her second substantial setback $(\mathrm{A}=2)$. Jamie reacted, "I know, it's wrong" while her answer was again graded as incorrect, suggesting some frustration. Her repeated incorrect answer initiated a new personalized feedback message, which said "Remember, this problem starts with a rectangle of 1/3, not 1 whole" (see Figure 2, center). This piece of medium-level scaffolded feedback was designed to make explicit the concept of a given area model that did not represent 1 whole.

Once more, Jamie responded resiliently upon receival of her feedback and decided to re-engage to make another new effort toward a task solution $(\mathrm{B}=2)$. Jamie re-initiated this new effort by pointing at the given rectangle and saying "Ohh, this is $1 / 3$ " and re-sustained her effort by sketching a rectangle that iterated two copies of the given rectangle of $1 / 3$ to create a rectangle with an area of 12 square units ( 2 units by 6 units) as her answer for 2/3. She submitted this answer and it was graded as correct (see Figure 2, right), thus this new effort was determined to be mathematically productive $(\mathrm{C}=1)$. Jamie celebrated by saying, "Yes! This is cool!" as the answer was graded as correct, suggesting her excitement and enjoyment with the Drawn2Math application. In sum, on Task I, Jamie encountered two substantial setbacks $(A=2)$, made two new efforts in response to those setbacks $(B=2)$, and one of those new efforts was mathematically productive $(\mathrm{C}=1)$. Thus, on Task I, her Reengagement Rate was $100 \%$ and her Perseverance Success Rate was $50 \%$. 
Next, consider Task II, which prompted Jamie to draw an area model of $1 / 6$ given a rectangular area model representing 1/3. The given model of $1 / 3$ had an area of 6 square units ( 2 units by 3 units). Reading the instructions silently, Jamie initiated and sustained her effort toward a task solution by quickly sketching a rectangle with an area of 2 square units ( 2 units by 1 unit) as her answer. It seemed she was sketching one-third of the given rectangle. She submitted this answer and it was graded as incorrect, marking her first substantial setback $(\mathrm{A}=1)$. Jamie reacted silently with her body language expressing disappointment. This initial incorrect answer again initiated a piece of lower-level scaffolded feedback designed to gently remind her refocus her attention on the given information. The feedback said, "The size of your 1/6 is incorrect" (see Figure 3, left). Jamie's mistake may have stemmed from understanding that $1 / 6$ is a smaller quantity than $1 / 3$, but not completely understanding the exact relationship between the size of sixths compared to thirds. Jamie responded resiliently to this setback and re-engaged to make a new effort toward a task solution $(\mathrm{B}=1)$. She re-initiated her new effort by deciding to explore some new ideas using the scratchwork pen. She re-sustained her new effort by sketching some circular area models depicting $2 / 6$ and $1 / 3$ (see Figure 3, center). She said, "I know these are the same," referring to the equivalence of $2 / 6$ and $1 / 3$. After studying her scratch work, Jamie drew a rectangle with an area of 2 square units (2 units by 1 unit) as her answer, which was the same answer as her previous incorrect submission. Despite Jamie's new efforts to explore these ideas using a different kind of visual representation, this new effort was determined to be not mathematically productive $(\mathrm{C}=0)$. She submitted this answer and it was again graded as incorrect, marking her second substantial setback $(\mathrm{A}=2)$. Jamie responded, "Yeah, I know but what else can I do?" to the notification of her incorrect answer, suggesting she was substantially unsure how to proceed with this task. At this point she received her second piece of personalized feedback, which said "The size of your 1/6 is too small" (see Figure 3, right). This piece of medium-level scaffolded feedback was designed to provoke the student to think about a more exact relationship between the size of sixths compared to thirds.

\section{FIGURE 3 \\ JAMIE'S EXPERIENCE ON TASK II}
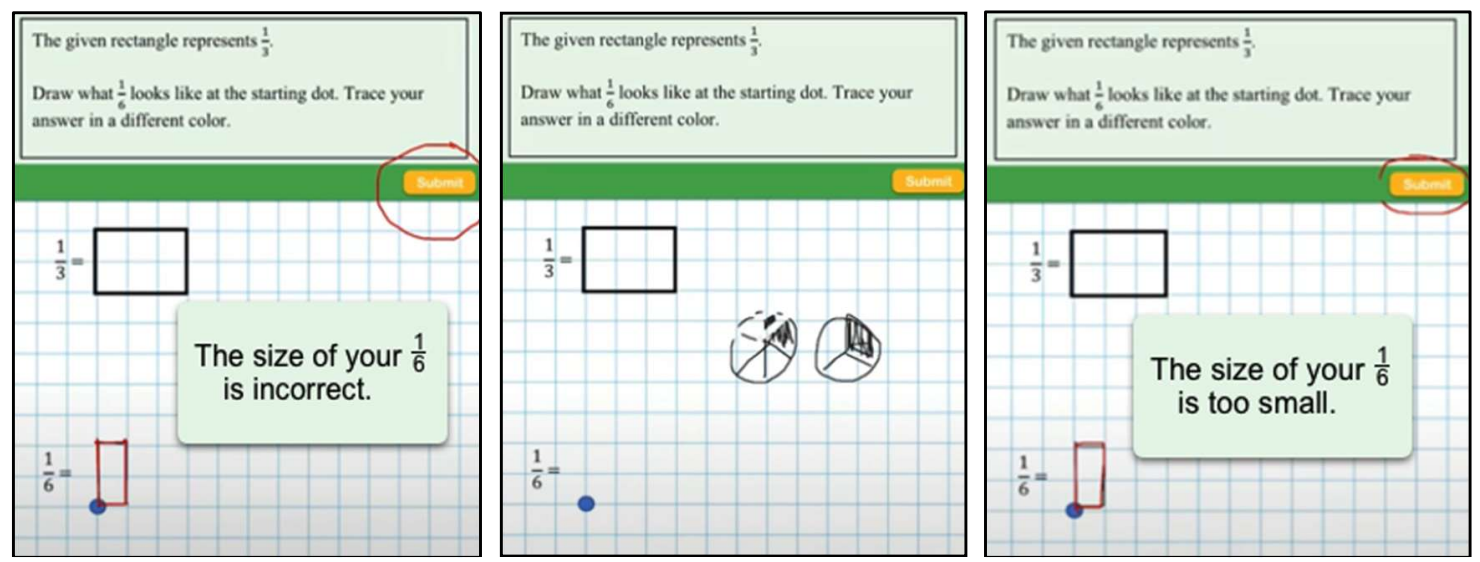

Jamie again responded resiliently to this feedback and re-engaged to make another new effort toward a task solution $(\mathrm{B}=2)$. Jamie re-initiated this new effort by again deciding to explore some new ideas using the scratch-work pen. She re-sustained her effort by sketching a copy of the given rectangle that represented $1 / 3$ and partitioning it into 6 equal pieces (see Figure 4, left). She narrated her work by saying, "I'm going to cut this up," referring to her partitioning work with the given model of $1 / 3$. After pondering her scratch work, Jamie sketched a rectangle with an area of 4 square units ( 2 units by 2 units) as her answer, which was incorrect. She said, "I know it's like half, this is close" as she submitted her answer. Despite the incorrect answer, these new efforts were determined to be mathematical productive $(\mathrm{C}=1)$ because Jamie was using her scratch work to think about how to use partitioned pieces of the given $1 / 3$ to build her model of $1 / 6$. Additionally, Jamie mentioned in her think-aloud that she knew that $1 / 6$ was one half the size of $1 / 3$, 
which was correct, yet for some reason she sketched a rectangle that was non-representative of that relationship. Thus, her submitted answer was graded as incorrect, marking her third substantial setback (A =3). Jamie reacted, "There is no other way," seemingly convinced that this task was not possible to solve. At this point she received her third piece of personalized feedback, which said "Try using a horizontal line to help you split up the given rectangle into smaller pieces" (see Figure 4, center). This piece of higherlevel scaffolded feedback was designed to suggest a practical problem-solving strategy that may have eluded the student thus far.

\section{FIGURE 4 JAMIE'S EXPERIENCE ON TASK II (CONTINUED)}
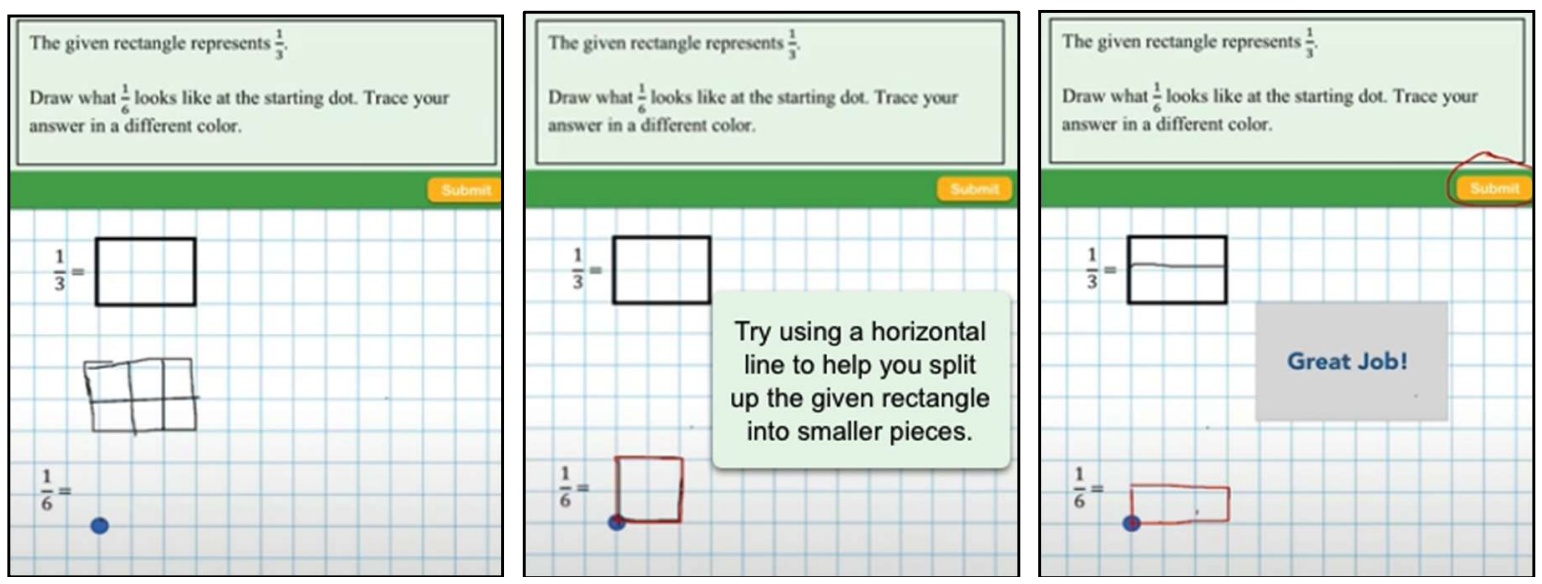

Jamie immediately and resiliently responded to this feedback by saying, "Ohh, I didn't know I could do that! I didn't know how to draw three boxes." Jamie's reaction provided some insight into her previous incorrect submission. It seemed that through Jamie's previous scratch work in which she partitioned the area of model of $1 / 3$ into 6 equal pieces, she came to understand that $1 / 3$ was equivalent to $2 / 6$, and half of $2 / 6$ would represent $1 / 6$, or 3 of the square-unit boxes on the digital page. However, a method of sketching $1 / 6$ as an area of 3 square units did not occur to Jamie in these moments, so she sketched 1/6 as an area of 4 square units instead, which she believed to be "close." After her reaction, Jamie re-engaged to make another new effort toward a task solution $(\mathrm{B}=3)$. She re-initiated this new effort by again deciding to use the scratch-work pen and re-sustained her new effort by partitioning the given model of $1 / 3$ into 2 equal pieces using a horizontal line. Jamie then sketched her new answer as a rectangle with an area of 3 square units ( 1 unit by 3 units). She submitted this answer and it was graded as correct (see Figure 4, right), thus this new effort was determined to be mathematically productive $(\mathrm{C}=2)$. Jamie exclaimed, "Yes!" to celebrate her correct answer, again suggesting her excitement and enjoyment with the Drawn2Math application. In sum, on Task II, Jamie encountered three substantial setbacks $(\mathrm{A}=3)$, made three new efforts in response to those setbacks $(\mathrm{B}=3)$, and two of those new efforts were mathematically productive $(\mathrm{C}=2)$. Thus, on Task II, her Reengagement Rate was $100 \%$ and her Perseverance Success Rate was approximately $67 \%$.

Last, consider Task III, which prompted Jamie to draw an area model of $1 / 8$ given a rectangular area model representing 3/4. The given model of $3 / 4$ had an area of 12 square units ( 2 units by 6 units). Jamie initiated her effort toward a task solution by deciding to use the scratch-work pen to explore some ideas. She sustained her effort by first partitioning the given area model of $3 / 4$ into 3 equal pieces, presumably to show that $3 / 4$ contains 3 pieces of size $1 / 4$. Next, Jamie iterated on the model of $3 / 4$ by drawing an additional piece of size $1 / 4$ on the end, creating a model of 1 whole. Then, Jamie partitioned her model of 1 whole into 8 equal sized pieces and labelled these pieces as $1 / 8$. After this substantial scratch work, Jamie sketched a rectangle with an area of 2 square units ( 2 units by 1 unit) as her answer to represent 1/8. She submitted this answer and it was graded as correct (see Figure 5). On Task III, Jamie never encountered any substantial 
setbacks, and thus, her work on Task III was not applicable to her overall Reengagement Rate or Perseverance Success Rate. We chose to share Jamie's experience on Task III to help readers understand the ways in which Jamie was learning about fraction concepts throughout her engagement with Drawn2Math sketching-based tasks. Her work on Task III helps show how Jamie came to understand the ideas of iterating and partitioning as a result of her perseverance with Tasks I and II, and was able to apply both of these ideas to solve Task III without a setback.

\section{FIGURE 5 JAMIE'S EXPERIENCE ON TASK III}

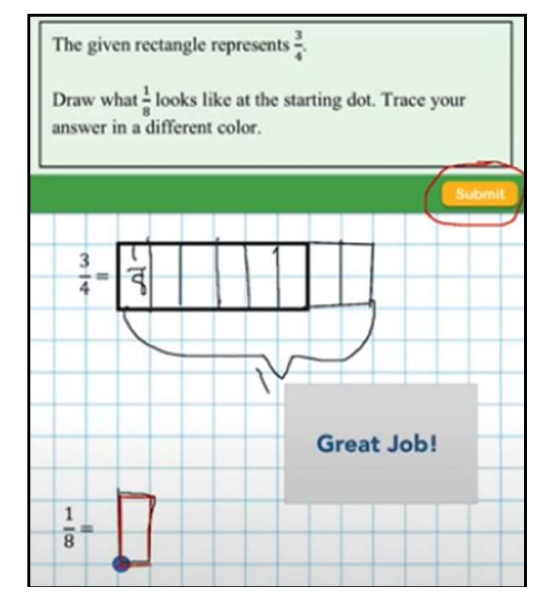

In all, Jamie's case is an apt representation of the entire Drawn2Math simulation dataset. Although we only reported about her experiences on three Drawn2Math sketching-based tasks, Jamie worked on a total of 18 tasks during her simulation and submitted incorrect sketches for 7 of them. In total, she encountered 11 substantial setbacks $(A=11)$, made 11 new efforts in response to those setbacks $(B=11)$, and 9 of those new efforts were mathematically productive $(\mathrm{C}=9)$. Thus, for her entire simulation, Jamie's Reengagement Rate was $100 \%$ and her Perseverance Success Rate was approximately $82 \%$. This is similar in comparison to the rates across all participants in the data set: a total Reengagement Rate of approximately $94 \%$ and a total Perseverance Success Rate of approximately $85 \%$. Jamie's detailed experiences help illustrate the primary outcomes of this work, namely that features of the Drawn2Math application helped participants stay engaged with mathematical tasks remotely, despite making mistakes, and participants could often leverage that engagement to persevere with challenging ideas to help develop mathematical understandings. Also, according to their think-aloud quotes, participants seemed to enjoy their time working on Drawn2Math sketching-based tasks and believed the feedback was helping them learn, despite encountering several setbacks along the way.

\section{CONCLUSIONS}

By simulating the important features of the Drawn2Math application, this study aimed to describe the efficacy of the application to remotely support student perseverance with sketching-based fractions tasks. Our findings showed that participants working on these challenging tasks were often able to leverage the personalized mathematical feedback to reengage and persevere with the task, even after substantial setbacks and experiencing ample frustration. All 10 participants showed evidence of this kind of success. Participants were reengaging and persevering with these sketching-based tasks despite working on them alone, remotely, without a teacher present. Participants even seemed to enjoy their work on these tasks, despite the many mistakes they made along the way. These findings suggest that mathematics education technology can help students stay engaged with challenging mathematics and learn conceptually, even when working remotely. 
One limitation of this study is that the Drawn2Math application features were being simulated by a remotely observing researcher. Thus, the next steps for this research will be to conduct a similar study using a functional application that uses an algorithm to provide the personalized feedback based upon student sketches. We will iterate on Drawn2Math to improve its sketch-recognition algorithm and feedback effectiveness, and will conduct a classroom trial to measure Drawn2Math's effect at a larger scale. This continued research will provide important contributions to advancing theory of how personalized feedback within a technology platform impacts perseverance in remote settings, and whether this will translate to conceptual learning - two important goals in the post-COVID19 education landscape.

\section{ACKNOWLEDGEMENT}

This study was supported by a grant from the National Science Foundation (Award Number 1831294).

\section{REFERENCES}

Anghileri, J. (2006). Scaffolding practices that enhance mathematics learning. Journal of Mathematics Teacher Education, 9(1), 33-52.

Arcavi, A. (2003). The role of visual representations in the learning of mathematics. Educational Studies in Mathematics, 52, 215-241.

Boaler, J., Chen, L., Williams, C., \& Cordero, M. (2016). Seeing as understanding: The importance of visual mathematics for our brain and learning. Journal of Applied Computational Mathematics, 5, 1-6.

Carver, C.S., \& Scheier, M.F. (2001). On the self-regulation of behavior. Cambridge, UK: Cambridge University Press.

DiNapoli, J. (2019a). "Getting better at sticking with it": Examining perseverance improvement in secondary mathematics students. In S. Otten, Z. de Araujo, A. Candela, C. Munter, \& C. Haines (Eds.), Proceedings of the 4lst Annual Meeting of the North American Chapter of the International Group for the Psychology of Mathematics Education (pp. 1386-1395). St. Louis, MO: University of Missouri.

DiNapoli, J. (2019b). Persevering toward what? Investigating the relationship between ninth-grade students' achievement goals and perseverant actions on an algebraic task. International Electronic Journal of Mathematics Education, 14(3), 435-453. DOI: https://doi.org/10.29333/iejme/5747

DiNapoli, J., \& Miller, E. K. (2020). Recognizing and supporting perseverance in mathematical problemsolving via conceptual thinking scaffolds. In M. Gresalfi \& I.S. Horn (Eds.), The Interdisciplinarity of the Learning Sciences, 14th International Conference of the Learning Sciences (ICLS) 2020, Volume 1 (pp. 11-18). Nashville, Tennessee: International Society of the Learning Sciences.

Freeburn, B., \& Arbaugh, F. (2017). Supporting productive struggle with communication moves. Mathematics Teacher, 111(3), 176-181.

Gresalfi, M.S., \& Barnes, J. (2015). Designing feedback in an immersive videogame: Supporting student mathematical engagement. Educational Technology Research and Development, 64(1), 1-22.

Hiebert, J., Carpenter, T.P., Fennema, E., Fuson, K., Human, P., Murray, H., . . Wearne, D. (1996). Problem solving as a basis for reform in curriculum and instruction: The case of mathematics. Educational Researcher, 25(4), 12-21.

Hubbard, E.M., Piazza, M., Pinel, P., \& Dehaene, S. (2005). Interactions between number and space in parietal cortex. Nature Reviews Neuroscience, 6(6), 435-448.

Kelley, J.F. (1984). An iterative design methodology for user-friendly natural language office information applications. ACM Transactions on Information Systems (TOIS), 2(1), 26-41.

Maher, C.A., \& Yankelewitz, D. (Eds.). (2017). Children's reasoning while building fraction ideas. New York, NY: Springer. 
Middleton, J.A., Tallman, M., Hatfield, N., \& Davis, O. (2015). Taking the severe out of perseverance: Strategies for building mathematical determination. In. N. Alpert (Ed.), The Collected Papers. Chicago, IL: Spencer Foundation.

Morales, H., Jr., \& DiNapoli, J. (2018). Latinx bilingual students' perseverance on a mathematical task: A rehumanizing perspective. REDIMAT: Journal of Research in Mathematics Education, 7(3), 226250.

National Council of Teachers of Mathematics (NCTM). (2014). Principles to Actions: Ensuring Mathematical Success for All. Reston, VA, NCTM.

National Research Council, \& Mathematics Learning Study Committee. (2001). Adding it up: Helping children learn mathematics. Washington, D.C.: National Academies Press.

Pólya, G. (1971). How to solve it (2nd ed.). Princeton: Princeton University Press.

Steffe, L.P., \& Olive, J. (2010). Children's fractional knowledge. New York, NY: Springer.

Zaslavsky, O. (2005). Seizing the opportunity to create uncertainty in learning mathematics. Educational Studies in Mathematics, 60, 297-321.

Zhang, M., Trussell, R.P., Gallegos, B., \& Asam, R.R. (2015). Using math apps for improving student learning: An exploratory study in an inclusive fourth grade classroom. TechTrends, 59(2), 32-39. 